\title{
Entanglement Classification for a Three-qubit System using Special Unitary Groups, SU(2) and SU(4)
}

\author{
Siti Munirah Mohd ${ }^{1}$ \\ Advanced Technology and Sustainability \\ Research Group PERMATA Insan College \\ Universiti Sains Islam Malaysia, Bandar Baru Nilai \\ 71800 Nilai, Negeri Sembilan, Malaysia \\ Bahari Idrus $^{2}$ \\ Center for Artificial Intelligence Technology \\ Faculty of Information Science and Technology \\ Universiti Kebangsaan Malaysia \\ 43600 Bangi, Selangor, Malaysia
}

\author{
Hishamuddin Zainuddin ${ }^{3}$ \\ Laboratory of Computational Sciences and Mathematical \\ Physics, Institute for Mathematical Research \\ University Putra Malaysia \\ 43400 UPM Serdang, Selangor, Malaysia
}

\author{
Muriati Mukhtar ${ }^{4}$ \\ Center for Software Technology and Management \\ Faculty of Information Science and Technology \\ Universiti Kebangsaan Malaysia \\ 43600 Bangi, Selangor, Malaysia
}

\begin{abstract}
Entanglement is a physical phenomenon that links a pair, or a set of particles that correlates with each other, regardless of the distance between them. Recent researches conducted on entanglement are mostly focused on measurement and classification in multiqubit systems. Classification of two qubits will only distinguish the quantum state as either separable or entangled, and it can be done by measurement. Meanwhile, in a three-qubit system, it becomes more complex because of the structure of the three qubits itself. It is not sufficient to do measurement because the states are divided into three types, including fully separable state, biseparable state, and genuine entangled state. Therefore, the classification is needed to distinguish the type of states in the three-qubit system. This study aims to classify the entanglement of three-qubit pure states using a combination model of special unitary groups, $S U(2)$ and $S U(4)$, by changing the angle of selected parameters in $S U(4)$ and acting on the separable pure state. The matrix represents $S U(2)$ is $2 \times 2$ matrix while matrix for $S U(4)$ is $4 \times 4$ matrix. Hence, the combination of $S U(2)$ and $S U(4)$ represent $8 \times 8$ matrix. This classification uses the von Neumann entropy and three tangle measurements to classify the class, respectively. The results of this study have indicated that the three-qubit pure state has been successfully classified into different classes, namely, $A-B-C, A-$ $B C, C-A B, G H Z$, and $W$, with $A-B-C$ being a fully separable state, $A-B C$ and $C-A B$ are biseparable states, and $G H Z$ and $W$ are genuine entangled states. The results show that this model can change separable pure states to other entanglement class after transformation is done.
\end{abstract}

Keywords-Quantum entanglement; multiqubit entanglement; entanglement classification; special unitary group; three-qubit system; quantum information

\section{INTRODUCTION}

The application of entanglement concept is known to be predominant in quantum computers. This research focuses on three qubits based on the knowledge that the structures of the qubits are more complex and applicable in actuality. This is due to the fact that in reality, quantum computers require more than three qubits to access data during high capacity information transmission. It is shown in the current recent, 20 qubits [1] and 50 qubits have been built and tested by IBM [2]. Throughout the time of entanglement concept's emergence, scientists initially rejected the idea because they were not convinced that entanglement could solve computer problem involving numerous data. However, entanglement became evident in the problem of quantum theory after Einstein, Podolsky, and Rosen (1935) [3-7] succeeded in describing the mysterious phenomenon inherent in quantum mechanics by initiating an experiment, known as the EPR paradox. Despite that, Einstein had doubted the concept of entanglement because the idea did not obey the limit of the speed of light. An assumption was made that the theory of quantum mechanics was incomplete at that time, supported by the fact that he made the second assumption about the existence of hidden variables which were yet to be found. It was also reported that Einstein's assumptions were proven wrong when John Bell [8] succeeded in proving the existence of entanglement in an experiment conducted in 1964 [3, 8, 9].

This research focuses on the classification of entanglement states in a multiqubit system [10-12] for the reason that normal measurements are unable to precisely differentiate the states. Previous research by Yang et al. [3] had only succeeded in classifying the quantum states into either separable, or entangled states due to the nature of structure in two qubits are simpler compared to the structure of multiqubit. Therefore, this research extends the inclusion of three qubits based on the fact that the classification of three qubits takes into consideration three types of state conditions known as fully separable, biseparable, and genuine entangled states [4, 13]. It is a known knowledge that the structure of the three-qubit system [14] is more complicated than the two-qubit system due to the additional parameters in each qubit. Furthermore, it is still equivocal whether the three-qubit structure can or cannot be transformed using local unitary. In order to classify the three-qubit state, various methods were used in the previous study such as the Generalized Schmidt Decomposition (GSD) [5], inductive method [6], special 
unitary group (SU), and special linear group (SL) [9, 11, 14, 15]. In spite of that, the classification of three-qubit systems using SU groups is not widely used at current, especially using $S U(2)$ and $S U(4)$. Although classification using $S U(2)$ has also been done on two qubits and three qubits, studies utilizing the $S U(2) \times S U(2) \times S U(2)$ combination, however only represent one qubit, which could only perform local operations for each qubit without affecting the other one. In addition, the six parameters used in previous study did not allow the selection of parameters to be done $[15,16]$. Nonetheless, a complete classification was made for selected simple cases, for example, the $2 \times 2 \times n . S U(4)$ system [17, 18] was found to be able to represent two qubits. That being said, the classification of the three-qubit system $[19,20]$ has not been performed using the combination of $S U(2)$ and $S U(4)$.

Therefore, based on the situations mentioned above, in this paper, the classification of three qubits is presented using $S U(2)$ and $S U(4)$ under local unitary transformation with selected parameters in specific angles from $S U(4)$. This study employs two types of measurements specifically to detect the state either entangled or separable during the classification. First, the von Neumann entropy $[19,21]$ is given as $S(\rho)=-\operatorname{Tr} \rho \log (\rho)$ and the second is the three-tangle [22] measure, $\tau_{3}$ or also known as the residue entanglement. Threetangle measure, $\tau_{3}$ is often used to differentiate between the $G H Z$-state and the $W$-state when these states become genuine entangled state $[23,24]$. The three-tangle measure used in this paper is given as, $\tau_{3}(\varphi)=4 \lambda_{0}^{2} \lambda_{4}^{2}$, with $\lambda$ representing the eigenvalues $[16,17]$.

\section{MAterial AND METHOD}

\section{A. Modeling Process}

This section explains the process of developing the combination model of $S U(2)$ and $S U(4)$ in details. The step by step process is implemented according to the four main steps as follows:

1) Understand the formula used and the range of angle for each parameter in $S U(2)$ parameterisation and $S U(4)$ parameterisation. The generator for each parameter used in $S U(2)$ and $S U(4)$ also has been studied.

2) Select six variable parameters from $S U(4)$ parameterisation based on the generator used with a fixed parameter used in $S U(2)$. The chosen generator was based on matrix characterisation, which is appropriate to enable the model to be developed. The selected parameters are combined using inner product operation. The range of angle for the parameter was based on the minimum and maximum range for an each of the parameter selected in $S U(4)$. Four combination angles from the range of angles of each parameter are fixed based on these lists:

- Both parameter angles are small, i.e., $\frac{\pi}{12}$.

- The first parameter angle is small, i.e., $\frac{\pi}{12}$ and the second parameter angle is large, i.e., $\frac{\pi}{2}$.
- The first parameter angle is large, i.e., $\frac{\pi}{2}$ and the second parameter angle is small, i.e., $\frac{\pi}{12}$

- Both parameter angles are large, i.e., $\frac{\pi}{2}$

3) Develop a matrix for the combination model of $S U(2)$ and $S U(4)$ operators, i.e., $S U(2) \times S U(4)$ and $S U(4) \times$ $S U(2)$. The operation between $S U(2)$ and $S U(4)$ is a tensor product between the parameter.

4) Develop the operator models $S U(2) \times S U(4)$ and $S U(4) \times S U(2)$, which are implemented on fully separable pure states. The process begins by first combining fully separable pure states with $S U(4) \times S U(2)$ and then combines with $S U(2) \times S U(4)$ to entangle the three qubits.

\section{B. SU(2) Parameterisation}

The $S U(2)$ parameterisation [19] is two complex dimensions that describe two level in the quantum system that represented with the $2 \times 2$ matrix. The $S U(2)$ parameterisation is given as,

$U=e^{i \sigma_{3} \beta_{1}} e^{i \sigma_{2} \beta_{2}} e^{i \sigma_{3} \beta_{3}}$

with $\beta_{1}, \beta_{2}, \beta_{3}$ representing parameters that satisfy the range of rotation angle, $0 \leq \beta_{1}, \beta_{3} \leq \pi$, and $0 \leq \beta_{2} \leq \frac{\pi}{2}$, with three generators, known as the Pauli matrix:

$\sigma_{1}=\left(\begin{array}{ll}0 & 1 \\ 1 & 0\end{array}\right), \sigma_{2}=\left(\begin{array}{cc}0 & -i \\ i & 0\end{array}\right), \sigma_{3}=\left(\begin{array}{cc}1 & 0 \\ 0 & -1\end{array}\right)$

\section{C. $S U(4)$ Parameterisation}

The $S U(4)$ parameterisation, as proposed by [18], is written as,

$$
\begin{aligned}
U= & e^{i \lambda_{3} \alpha_{1}} e^{i \lambda_{2} \alpha_{2}} e^{i \lambda_{3} \alpha_{3}} e^{i \lambda_{5} \alpha_{4}} e^{i \lambda_{3} \alpha_{5}} e^{i \lambda_{10} \alpha_{6}} e^{i \lambda_{3} \alpha_{7}} e^{i \lambda_{2} \alpha_{8}} e^{i \lambda_{3} \alpha_{9}} e^{i \lambda_{5} \alpha_{10}} e^{i \lambda_{3} \alpha_{11}} \\
& e^{i \lambda_{2} \alpha_{12}} e^{i \lambda_{3} \alpha_{13}} e^{i \lambda_{8} \alpha_{14}} e^{i \lambda_{12} \alpha_{15}}
\end{aligned}
$$

with $\lambda_{i}, i=1,2, \ldots, 15$ representing the generators. Parameters $\alpha_{1}$ until $\alpha_{15}$ are rotation angles of qubits in Hilbert space that satisfy the range:

$$
\begin{aligned}
& 0 \leq \alpha_{1}, \alpha_{7}, \alpha_{11} \leq \pi \\
& 0 \leq \alpha_{3}, \alpha_{5}, \alpha_{9}, \alpha_{13} \leq 2 \pi \\
& 0 \leq \alpha_{2}, \alpha_{4}, \alpha_{6}, \alpha_{8}, \alpha_{10}, \alpha_{12} \leq \frac{\pi}{2} \\
& 0 \leq \alpha_{14} \leq \sqrt{3} \pi \\
& 0 \leq \alpha_{15} \leq 2 \sqrt{\frac{2}{3}} \pi
\end{aligned}
$$

\section{Selection of Parameter}

This study selected two parameters from operator $S U(4)$ based on the reasoning that they can affect two qubits that are correlated to each other. The two chosen parameters were a combination of six selected parameters, namely; $\alpha_{2}, \alpha_{4}, \alpha_{6}, \alpha_{8}, \alpha_{10} \& \alpha_{12}$ based on the $\lambda_{2}, \lambda_{5} \& \lambda_{10}$ generators with selected angles. Some parameters were not chosen in $S U$ (2) since they do not influence other qubits. Besides, the qubit in $S U(2)$ did not have any correlation to the parameters 
in $S U$ (4). Parameters that used generators $\lambda_{3}, \lambda_{8}, \lambda_{15}$ were not considered since the solution was trivial. Constant parameters were fixed with the value of 0 to ensure that each parameter represented a real number.

\section{E. Matrix Development of Combination Model SU(2) $\times$ $S U(4)$ and $S U(4) \times S U(2)$}

This section describes the process of developing the matrix for models $S U(2) \times S U(4)$ and $S U(4) \times S U(2)$. Figure 1 illustrates the three main steps to develop the matrix.

1) Matrix Development for $\mathrm{SU}(2)$ and $\mathrm{SU}(4)$

Matrix multiplication for $S U(2)$ as an example for $e^{i \sigma_{3} \beta_{1}}$ :

$$
\begin{aligned}
e^{i \sigma_{3} \beta_{1}} & =I+i \sigma_{3} \beta_{1}+\frac{\left(i \sigma_{3} \beta_{1}\right)^{2}}{2 !}+\frac{\left(i \sigma_{3} \beta_{1}\right)^{3}}{3 !}+\frac{\left(i \sigma_{3} \beta_{1}\right)^{4}}{4 !}+\cdots \\
& =\cos \beta_{1} I+i \sin \beta_{1} \sigma_{3} \\
& =\cos \beta_{1}\left(\begin{array}{ll}
1 & 0 \\
0 & 1
\end{array}\right)+i \sin \beta_{1}\left(\begin{array}{cc}
1 & 0 \\
0 & -1
\end{array}\right) \\
& =\left(\begin{array}{cc}
\cos \beta_{1}+i \sin \beta_{1} & 0 \\
0 & \cos \beta_{1}-i \sin \beta_{1}
\end{array}\right)
\end{aligned}
$$
$e^{i \alpha_{2} \lambda_{2}}$

and matrix multiplication for $S U(4)$ as an example for

using generator

$$
\lambda_{2}=\left(\begin{array}{cccc}
0 & -i & 0 & 0 \\
i & 0 & 0 & 0 \\
0 & 0 & 0 & 0 \\
0 & 0 & 0 & 0
\end{array}\right)
$$

$$
\begin{aligned}
e^{i \alpha_{2} \lambda_{2}} & =I+i \alpha_{2} \lambda_{2}+\frac{\left(i \alpha_{2} \lambda_{2}\right)^{2}}{2 !}+\frac{\left(i \alpha_{2} \lambda_{2}\right)^{3}}{3 !}+\frac{\left(i \alpha_{2} \lambda_{2}\right)^{4}}{4 !}+\cdots \\
& =\left(I-\frac{\alpha_{2}^{2}}{2 !}+\frac{\alpha_{2}^{4}}{4 !}-\cdots\right) \lambda_{2}^{2}+\left(i \alpha_{2}-\frac{i \alpha_{2}^{3}}{3 !}+\frac{i \alpha_{2}^{5}}{5 !}+\cdots\right) \lambda_{2} \\
& =\left(\begin{array}{cccc}
\cos \alpha_{2} & \sin \alpha_{2} & 0 & 0 \\
-\sin \alpha_{2} & \cos \alpha_{2} & 0 & 0 \\
0 & 0 & 1 & 0 \\
0 & 0 & 0 & 1
\end{array}\right)
\end{aligned}
$$

In this paper, other exponents that were calculated the same as equation (6) were $e^{i \alpha_{2} \lambda_{2}}, e^{i \alpha_{8} \lambda_{2}}, e^{i \alpha_{12} \lambda_{2}}, e^{i \alpha_{4} \lambda_{5}}, e^{i \alpha_{10} \lambda_{5}}, e^{i \alpha_{6} \lambda_{10}}$

Matrix development of $S U(2)$ and $S U(4)$

Matrix development of inner product for two parameters in $S U(4)$

Matrix development of tensor product

for models $S U(2) X S U(4)$ and $S U(4) X S U(2)$

Fig. 1. Steps of Matrix Development

\section{2) Matrix Development for Two Parameters in SU(4)}

From the six selected parameters, two parameters were combined to produce 30 combinations of parameters, as shown in Table I.

An example of matrix multiplication for parameters $\alpha_{2}$ and $\alpha_{4}$ from two matrixes is as follows, $e^{i \alpha_{2} \lambda_{2}} \cdot e^{i \alpha_{4} \lambda_{5}}$

$$
\begin{aligned}
& e^{i \alpha_{2} \lambda_{2}} \cdot e^{i \alpha_{4} \lambda_{5}} \\
= & \left(\begin{array}{cccc}
\cos \alpha_{2} & \sin \alpha_{2} & 0 & 0 \\
-\sin \alpha_{2} & \cos \alpha_{2} & 0 & 0 \\
0 & 0 & 1 & 0 \\
0 & 0 & 0 & 1
\end{array}\right)\left(\begin{array}{cccc}
\cos \alpha_{4} & 0 & \sin \alpha_{4} & 0 \\
0 & 1 & 0 & 0 \\
-\sin \alpha_{4} & 0 & \cos \alpha_{4} & 0 \\
0 & 0 & 0 & 1
\end{array}\right) \\
= & \left(\begin{array}{cccc}
\cos \alpha_{2} \cos \alpha_{4} & \sin \alpha_{2} & \cos \alpha_{2} \sin \alpha_{4} & 0 \\
-\cos \alpha_{4} \sin \alpha_{2} & \cos \alpha_{2} & -\sin \alpha_{2} \sin \alpha_{4} & 0 \\
-\sin \alpha_{4} & 0 & \cos \alpha_{4} & 0 \\
0 & 0 & 0 & 1
\end{array}\right)
\end{aligned}
$$

For matrix development of the tensor product for $S U(2) \times$ $S U(4)$ and $S U(4) \times S U(2)$, an example for the parameter $\alpha_{2}$ $\alpha_{4}$ with matrix identity, $I_{2}$ for $S U(2) \times S U(4)$ in equation (8). The calculation for $S U(4) \times S U(2)$ in equation (9) using same the parameter of $\alpha_{2} \alpha_{4}$ with matrix identity, $I_{2}$.

$$
\begin{aligned}
& S U(2) \times S U(4) \\
& \left(\begin{array}{cccccccc}
\cos \alpha_{2} \cos \alpha_{4} & \sin \alpha_{2} & \cos \alpha_{2} \sin \alpha_{4} & 0 & 0 & 0 & 0 & 0 \\
-\cos \alpha_{4} \sin \alpha_{2} & \cos \alpha_{2} & -\sin \alpha_{2} \sin \alpha_{4} & 0 & 0 & 0 & 0 & 0 \\
-\sin \alpha_{4} & 0 & \cos \alpha_{4} & 0 & 0 & 0 & 0 & 0 \\
0 & 0 & 0 & 1 & 0 & 0 & 0 & 0 \\
0 & 0 & 0 & 0 & \cos \alpha_{2} \cos \alpha_{4} & \sin \alpha_{2} & \cos \alpha_{2} \sin \alpha_{4} & 0 \\
0 & 0 & 0 & 0 & -\cos \alpha_{4} \sin \alpha_{2} & \cos \alpha_{2} & -\sin \alpha_{2} \sin \alpha_{4} & 0 \\
0 & 0 & 0 & 0 & -\sin \alpha_{4} & 0 & \cos \alpha_{4} & 0 \\
0 & 0 & 0 & 0 & 0 & 0 & 0 & 1
\end{array}\right)
\end{aligned}
$$

$\operatorname{SU}(4) \times S U(2)=$

$\left(\begin{array}{cccccccc}\cos \alpha_{2} \cos \alpha_{4} & 0 & \sin \alpha_{2} & 0 & \cos \alpha_{2} \sin \alpha_{4} & 0 & 0 & 0 \\ 0 & \cos \alpha_{2} \cos \alpha_{4} & 0 & \sin \alpha_{2} & 0 & \cos \alpha_{2} \sin \alpha_{4} & 0 & 0 \\ -\cos \alpha_{4} \sin \alpha_{2} & 0 & \cos \alpha_{2} & 0 & -\sin \alpha_{2} \sin \alpha_{4} & 0 & 0 & 0 \\ 0 & -\cos \alpha_{4} \sin \alpha_{2} & 0 & \cos \alpha_{2} & 0 & -\sin \alpha_{2} \sin \alpha_{4} & 0 & 0 \\ -\sin \alpha_{4} & 0 & 0 & 0 & \cos \alpha_{4} & 0 & 0 & 0 \\ 0 & -\sin \alpha_{4} & 0 & 0 & 0 & \cos \alpha_{4} & 0 & 0 \\ 0 & 0 & 0 & 0 & 0 & 0 & 1 & 0 \\ 0 & 0 & 0 & 0 & 0 & 0 & 0 & 1\end{array}\right)$

The remaining parameters in Table I has been developed by the same multiplication in equation (8) and equation (9) using MATHEMATICA software.

\section{F. Operating Flow Chart of Combination Model on Fully Separable Three Qubit Pure State}

Figure 2 shows the flowchart of notation $U 42$ being used to represent $S U(4) \times S U(2)$ and $U 24$ for $S U(2) \times S U(4)$ on fully separable three-qubit pure states. The fully separable three-qubit pure states are represented by $|000\rangle$ and the time interval used is every 10 second. 
TABLE. I. COMBINATIONS OF TWO PARAMETERS IN $\boldsymbol{S} \boldsymbol{U}(\mathbf{4})$

\begin{tabular}{|l|l|l|l|l|l|l|}
\hline No. & Combination & No. & Combination & & No. & Combination \\
\hline 1. & $\alpha_{2}, \alpha_{4}$ & 11. & $\alpha_{6}, \alpha_{2}$ & & 21. & $\alpha_{10}, \alpha_{2}$ \\
\hline 2. & $\alpha_{2}, \alpha_{6}$ & 12. & $\alpha_{6}, \alpha_{4}$ & & 22. & $\alpha_{10}, \alpha_{4}$ \\
\hline 3. & $\alpha_{2}, \alpha_{8}$ & 13. & $\alpha_{6}, \alpha_{8}$ & 23. & $\alpha_{10}, \alpha_{6}$ \\
\hline 4. & $\alpha_{2}, \alpha_{10}$ & 14. & $\alpha_{6}, \alpha_{10}$ & 24. & $\alpha_{10}, \alpha_{8}$ \\
\hline 5. & $\alpha_{2}, \alpha_{12}$ & 15. & $\alpha_{6}, \alpha_{12}$ & 25. & $\alpha_{10}, \alpha_{12}$ \\
\hline 6. & $\alpha_{4}, \alpha_{2}$ & 16. & $\alpha_{8}, \alpha_{2}$ & 26. & $\alpha_{12}, \alpha_{2}$ \\
\hline 7. & $\alpha_{4}, \alpha_{6}$ & 17. & $\alpha_{8}, \alpha_{4}$ & 27. & $\alpha_{12}, \alpha_{4}$ \\
\hline 8. & $\alpha_{4}, \alpha_{8}$ & 18. & $\alpha_{8}, \alpha_{6}$ & & 28. & $\alpha_{12}, \alpha_{6}$ \\
\hline 9. & $\alpha_{4}, \alpha_{10}$ & 19. & $\alpha_{8}, \alpha_{10}$ & 29. & $\alpha_{12}, \alpha_{8}$ \\
\hline 10. & $\alpha_{4}, \alpha_{12}$ & 20. & $\alpha_{8}, \alpha_{12}$ & 30. & $\alpha_{12}, \alpha_{10}$ \\
\hline
\end{tabular}

The step by step flowchart in Figure 2 is described as follows:

1) Evolution started at $t=0 \mathrm{~s}$, with $U 42|000\rangle$ and the first combination of parameters $\alpha_{2}, \alpha_{4}$, with the angle of $\alpha_{2}=\frac{\pi}{12}$ and $\alpha_{4}=\frac{\pi}{12}$

2) ${ }^{12}$ At $t=10 s,{ }^{12} U 24|\psi\rangle$ began to evolve until $t=20 \mathrm{~s}$, with the same angle in step (i). The newly obtained state was measured using von Neumann entropy and three tangle measurements, which was classified in the list given in Table II. Range value for the von Neumann entropy is $0 £ S(r) £ 1$ for all values of the density matrix, $r$ with zero value indicating a separable state and one indicating maximum entanglement. For the three-tangle measurement, zero value indicated the $W$-state, while a positive value indicated the GHZ state.

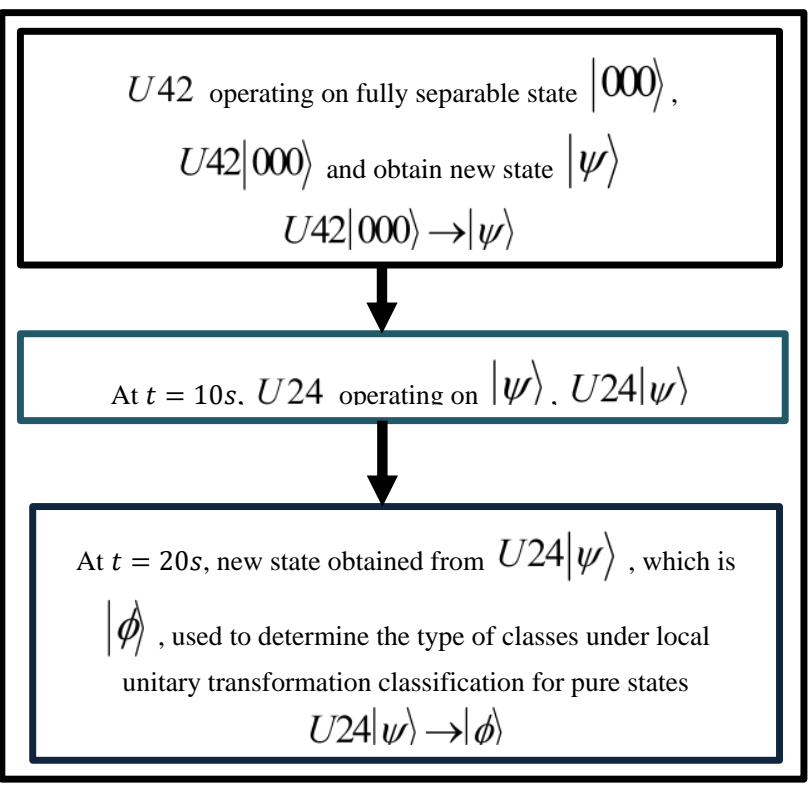

Fig. 2. Operating $S U(2) \times S U(4)$ and $S U(4) \times S U(2)$ on Fully Separable States.
TABLE. II. VON NEUMANN ENTROPy VALUE AND ThreE-TANGLE MEASUREMENT

\begin{tabular}{|l|c|c|c|c|}
\hline \multicolumn{1}{|c|}{ Class } & $\begin{array}{l}\text { Qubit A, } \\
S_{A}\end{array}$ & $\begin{array}{l}\text { Qubit B, } \\
S_{B}\end{array}$ & $\begin{array}{l}\text { Qubit C, } \\
S_{C}\end{array}$ & $\begin{array}{l}\text { Three } \\
\text { tangle, } \tau_{3}\end{array}$ \\
\hline$A-B-C$ & 0 & 0 & 0 & 0 \\
\hline$A-B C$ & 0 & $>0$ & $>0$ & 0 \\
\hline$B-A C$ & $>0$ & 0 & $>0$ & 0 \\
\hline$C-A B$ & $>0$ & $>0$ & 0 & 0 \\
\hline$W$ & $>0$ & $>0$ & $>0$ & 0 \\
\hline$G H Z$ & $>0$ & $>0$ & $>0$ & $>0$ \\
\hline
\end{tabular}

3) Steps (i) and (ii) were repeated with three other angles for the same parameters' combination:

$$
\begin{aligned}
& \alpha_{2}=\frac{\pi}{12} \text { and } \alpha_{4}=\frac{\pi}{2} \\
& \alpha_{2}=\frac{\pi}{2} \text { and } \alpha_{4}=\frac{\pi}{12} \\
& \alpha_{2}=\frac{\pi}{2} \text { and } \alpha_{4}=\frac{\pi}{2}
\end{aligned}
$$

4) Step (i) until (iii) were repeated for all parameters combination, as shown in Table I.

\section{RESUltS AND ANALYSIS}

The results from the operation of the model to the fully separable states were analysed based on the patterns of von Neumann entropy and values shown in Table II.

\section{A. Patterns of von Neumann Entropy, Before and After the Operation with $S U(2) X S U(4)$}

Table III presents the categories for the 30 parameter combinations, which are divided into eight groups, as determined by pattern values of the von Neumann entropy in each combination of parameters in Table I.

\section{B. Classification of Fully Separable Three-qubit Pure State Following Operation by the Model of the Operator $S U(2) \times S U(4)$}

The results of this study have indicated that the three-qubit pure state has been successfully classified into different classes, namely; $A-B-C, A-B C, C-A B, G H Z$, and $W$, with $A-B-C$ being a fully separable state, $A-B C$ and $C-A B$ are biseparable states, and $G H Z$ and $W$ are genuine entangled states. Prior to being combined with $S U(2) \times S U(4)$, the three-qubit pure state could only exist in two types of classes, namely, (i) fully separable state $(A-B-C)$ and (ii) biseparable state $(C-A B)$. After the combination with $S U(2) \times S U(4)$, the state is turned into five classes, namely, $A-B-C, A-B C, C-A B, G H Z$, and $W$. At combination angles of $\frac{\pi}{2} \& \frac{\pi}{2}$, the state of the three-qubit is classified into $A-B-C$, before and after being combined 
with $S U(2) \times S U(4)$. The combination angles $\frac{\pi}{12} \& \frac{\pi}{12}$ transformed the state from fully separable to genuine entangled state for all groups, except for group 3 and group 7. Group 7 gives a classification class of fully separable states for any combination angles of parameters. Table IV summarises the types of classifications for the pure state of three qubits, before and after being combined with $S U(2) \times S U$ (4) comprising eight groups and four types of combined angles.

TABLE. III. GROUPS OF PARAMETERS COMBINATION

\begin{tabular}{|l|l|}
\hline Group & Parameters combination \\
\hline 1 & $\alpha_{4}, \alpha_{2}, \alpha_{4}, \alpha_{8}, \alpha_{4}, \alpha_{12}, \alpha_{10}, \alpha_{2}, \alpha_{10}, \alpha_{8}, \alpha_{10}, \alpha_{12}$ \\
\hline 2 & $\alpha_{2}, \alpha_{4}, \alpha_{2}, \alpha_{10}, \alpha_{8}, \alpha_{4}, \alpha_{8}, \alpha_{10}, \alpha_{12}, \alpha_{4}, \alpha_{12}, \alpha_{10}$ \\
\hline 3 & $\alpha_{2}, \alpha_{8}, \alpha_{2}, \alpha_{12}, \alpha_{8}, \alpha_{2}, \alpha_{8}, \alpha_{12}, \alpha_{12}, \alpha_{2}, \alpha_{12}, \alpha_{8}$ \\
\hline 4 & $\alpha_{6}, \alpha_{2}, \alpha_{6}, \alpha_{8}, \alpha_{6}, \alpha_{12}$ \\
\hline 5 & $\alpha_{6}, \alpha_{4}, \alpha_{6}, \alpha_{10}$ \\
\hline 6 & $\alpha_{2}, \alpha_{6}, \alpha_{8}, \alpha_{6}, \alpha_{12}, \alpha_{6}$ \\
\hline 7 & $\alpha_{4}, \alpha_{10}, \alpha_{10}, \alpha_{4}$ \\
\hline 8 & $\alpha_{4}, \alpha_{6}, \alpha_{10}, \alpha_{6}$ \\
\hline
\end{tabular}

TABLE. IV. Classifications of Three-Qubit Pure State Before AND AFTER COMBINATION WITH $S U(2) \times S U(4)$

\begin{tabular}{|c|c|c|c|c|c|c|c|c|}
\hline \multirow{2}{*}{$\begin{array}{l}\text { Angle } \\
\text { Group }\end{array}$} & \multicolumn{4}{|c|}{$\begin{array}{c}\text { BEFORE COMBINATION WITH } \\
S U(2) \times S U(4)\end{array}$} & \multicolumn{4}{|c|}{$\begin{array}{l}\text { AFTER COMBINATION WITH } \\
\qquad S U(2) \times S U(4)\end{array}$} \\
\hline & $\frac{\pi}{12} \& \frac{\pi}{12}$ & $\frac{\pi}{12} \& \frac{\pi}{2}$ & $\frac{\pi}{2} \& \frac{\pi}{12}$ & $\frac{\pi}{2} \& \frac{\pi}{2}$ & $\frac{\pi}{12} \& \frac{\pi}{12}$ & $\frac{\pi}{12} \& \frac{\pi}{2}$ & $\frac{\pi}{2} \& \frac{\pi}{12}$ & $\frac{\pi}{2} \& \frac{\pi}{2}$ \\
\hline 1 & $\mathrm{C}-\mathrm{AB}$ & $\begin{array}{l}\text { A-B- } \\
\text { C }\end{array}$ & C-AB & $\begin{array}{l}\text { A-B- } \\
\text { C }\end{array}$ & GHZ & $\begin{array}{l}\text { A-B- } \\
\text { C }\end{array}$ & W & A-B-C \\
\hline 2 & $\mathrm{C}-\mathrm{AB}$ & $\begin{array}{l}\text { A-B- } \\
\text { C }\end{array}$ & C-AB & $\begin{array}{l}\text { A-B- } \\
\text { C }\end{array}$ & GHZ & $\begin{array}{l}\text { A-B- } \\
\text { C }\end{array}$ & GHZ & A-B-C \\
\hline 3 & A-B-C & $\begin{array}{l}\text { A-B- } \\
\text { C }\end{array}$ & $\begin{array}{l}\text { A-B- } \\
\text { C }\end{array}$ & $\begin{array}{l}\text { A-B- } \\
\text { C }\end{array}$ & $\mathrm{A}-\mathrm{BC}$ & A-BC & A-BC & A-B-C \\
\hline 4 & $\mathrm{C}-\mathrm{AB}$ & $\begin{array}{l}\text { A-B- } \\
\text { C }\end{array}$ & $\begin{array}{l}\text { A-B- } \\
\text { C }\end{array}$ & $\begin{array}{l}\text { A-B- } \\
\text { C }\end{array}$ & GHZ & $\begin{array}{l}\text { A-B- } \\
\text { C }\end{array}$ & $\begin{array}{l}\text { A-B- } \\
\text { C }\end{array}$ & A-B-C \\
\hline 5 & $\mathrm{C}-\mathrm{AB}$ & $\begin{array}{l}\text { A-B- } \\
\text { C }\end{array}$ & $\begin{array}{l}\text { A-B- } \\
\text { C }\end{array}$ & $\begin{array}{l}\text { A-B- } \\
\text { C }\end{array}$ & $\mathrm{W}$ & $\begin{array}{l}\text { A-B- } \\
\text { C }\end{array}$ & $\begin{array}{l}\text { A-B- } \\
\text { C }\end{array}$ & A-B-C \\
\hline 6 & $\mathrm{C}-\mathrm{AB}$ & $\begin{array}{l}\text { A-B- } \\
\text { C }\end{array}$ & $\begin{array}{l}\text { A-B- } \\
\text { C }\end{array}$ & $\begin{array}{l}\text { A-B- } \\
\text { C }\end{array}$ & GHZ & $\begin{array}{l}\text { A-B- } \\
\text { C }\end{array}$ & $\begin{array}{l}\text { A-B- } \\
\text { C }\end{array}$ & A-B-C \\
\hline 7 & A-B-C & $\begin{array}{l}\text { A-B- } \\
\text { C }\end{array}$ & $\begin{array}{l}\text { A-B- } \\
\text { C }\end{array}$ & $\begin{array}{l}\text { A-B- } \\
\text { C }\end{array}$ & A-B-C & $\begin{array}{l}\text { A-B- } \\
\text { C }\end{array}$ & $\begin{array}{l}\text { A-B- } \\
\text { C }\end{array}$ & A-B-C \\
\hline 8 & $\mathrm{C}-\mathrm{AB}$ & $\begin{array}{l}\text { A-B- } \\
\text { C }\end{array}$ & $\begin{array}{l}\text { A-B- } \\
\text { C }\end{array}$ & $\begin{array}{l}\text { A-B- } \\
\text { C }\end{array}$ & GHZ & $\begin{array}{l}\text { A-B- } \\
\text { C }\end{array}$ & A-BC & A-B-C \\
\hline
\end{tabular}

\section{Discussion of the Result}

The classification using combinations of special unitary groups, $S U(2)$ and $S U(4)$ is based on three cases of selection on angle in $S U(4)$ parameters which are (i) both angles is small, (ii) one angle is small and another one is big, and (iii) both angle are big. In theory, this study aid in calculating the entanglement that involves three-qubit with considering the changing of the angle at the two-qubit that already correlate in the early combination. By determine the angle, it will make the classification of three-qubit more easy without considering all angle in the early combination. Hence, this study can be extended to the complex state since the state used that is fully separable is the simplest state. Besides, by changing the angle of the parameter, the researcher can fix in the beginning of the process to get the desired result.

\section{CONCLUSIONS}

This study has shown that classification using combinations of special unitary groups, $S U(2)$ and $S U(4)$, can be conducted according to the types of the class under the local unitary transformation. It is also found in this study that the three qubits, with fully separable states, can be classified into fully separable states $(A-B-C)$, biseparable states $(A-B C, C-A B), G H Z$-like state, and $W$-like state. This classification has been achieved by considering two main aspects, namely, the group of the parameter's combination in $S U$ (4) and the range of combination angles of the two parameters. Class $A-B-C$ was obtained from group 7, with combination angles of $\frac{\pi}{12} \& \frac{\pi}{2}$, and $\frac{\pi}{2} \& \frac{\pi}{2}$, before and after their combination with the $S U(2) \times S U(4)$ operator. Class $A-B C$ was obtained after the operator, $S U(2) \times S U(4)$ from group 3, was combined at combination angles of $\frac{\pi}{12} \& \frac{\pi}{12}, \frac{\pi}{12} \& \frac{\pi}{2}$, and $\frac{\pi}{2} \& \frac{\pi}{12}$, while from group 8 , the operator was combined at combination angles of $\frac{\pi}{2} \& \frac{\pi}{12}$. Class $C-A B$ was achieved at combination angles of $\frac{\pi}{12} \& \frac{\pi}{12}$ and $\frac{\pi}{2} \& \frac{\pi}{12}$ before being combined with operator $S U(2) \times S U(4)$. In the combination angles $\frac{\pi}{12} \& \frac{\pi}{12}$ from group $1,2,4,6$, and 8 , the results gave $G H Z$-like state, while $W$-like state was obtained for groups 1 and 5 at combination angles of $\frac{\pi}{2} \& \frac{\pi}{12}$ and $\frac{\pi}{12} \& \frac{\pi}{12}$, respectively. These findings have significant implications in understanding how the $S U(2) \times S U(4)$ and $S U(4) \times S U(2)$ operators play the role of transforming the initial state into other classes by only controlling the angle of the parameter in $S U(4)$. With respect to the fact that this study is only limited to fully separable states and does not classify the $B-A C$ class from the simulation, further work needs to be done by considering other parameters combination in $S U(4)$ and other ranges of parameter angles that inlvove real and imaginary value, including changing fully separable states into biseparable states to upgrade the model hence the algorithm become simpler and can be tested in other class of state. Besides, this research also can be extended by combining three parameters that will be changing to see the outcome of the types of class occur. 


\section{ACKNOWLEDGMENT}

We would like to express our gratitude to the Ministry of Higher Education Malaysia for the research grants, Code: FGRS/1/2011/SG/UKM/03/5 and ERGS/1/2013/ICT 07/UKM/02/2 that enabled us to come up with this research.

\section{REFERENCES}

[1] J. Mooney, G., C. D. Hill, and L. C. L. Hollenberg, Entanglement in a 20-Qubit Superconducting Quantum Computer. 2019.

[2] Dimić, A. and B. Dakić, Single-copy entanglement detection. npj Quantum Information, 2018. 4(1): p. 11.

[3] Yang, X.-G., et al., Classification of Bipartite and Tripartite Qutrit Entanglement Under SLOCC. Communications in Theoretical Physics, 2008. 50(3): p. 651.

[4] Allan, I.S. and H. Choon-Lin, Condition for tripartite entanglement. Journal of Physics: Conference Series, 2012. 343(1): p. 012114.

[5] Acín, A., et al., Generalized Schmidt Decomposition and Classification of Three-Quantum-Bit States. Physical Review Letters, 2000. 85(7): p. 1560-1563.

[6] Lamata, L., et al., Inductive classification of multipartite entanglement under stochastic local operations and classical communication. Physical Review A, 2006. 74(5): p. 052336.

[7] Peres, A., Einstein, Podolsky, Rosen, and Shannon. Foundations of Physics, 2005. 35(3): p. 511-514.

[8] Aspect, A., P. Grangier, and G. Roger, Experimental Tests of Realistic Local Theories via Bell's Theorem. Physical Review Letters, 1981. 47(7): p. 460-463.

[9] Osterloh, A., Classification of qubit entanglement: $\operatorname{SL}(2, \mathbb{C})$ versus $\mathrm{SU}(2)$ invariance. Applied Physics B, 2010. 98(4): p. 609-616.

[10] Siti Munirah, M., et al., Measurement for Multipartite Entanglement: Detection and Quantification, in Regional conference on science, technology and social sciences. 2014: Cameron Highlands, Pahang.

[11] Li, J.-L., S.-Y. Li, and Q. Cong-Feng, Classification of the Entangled States of \$2ltimes Lltimes Mltimes Nltimes H\$. Vol. 85. 2011.
[12] Akhound, A., S. Haddadi, and M.A.C. Motlagh, Analyzing the entanglement properties of graph states with generalized concurrence. Modern Physics Letters B, 2019. 33(10): p. 1950118.

[13] Siti Munirah Mohd, et al., A Framework of Methods Classification for Multipartite Entanglement. Journal of Theoretical and Applied Information Technology, 2015. 81(3): p. 438-445.

[14] Sun, L.-L., J.-L. Li, and C.-F. Qiao, Classification of the entangled states of $\$ \$ 2 \backslash$ times $L$ \times $M \backslash$ times $N \$ \$ 2 \times L \times M \times N$. Quantum Information Processing, 2015. 14(1): p. 229-245.

[15] Liu, B., et al., Local Unitary Classification of Arbitrary Dimensional Multipartite Pure States. Physical Review Letters, 2012. 108(5): p. 050501.

[16] Ryszard, H., et al., Quantum entanglement. Reviews of Modern Physics, 2009. 81(2): p. 865-942.

[17] Coffman, V., J. Kundu, and W.K. Wootters, Distributed entanglement. Physical Review A, 2000. 61(5): p. 052306.

[18] Todd, T., B. Mark, and E.C.G. Sudarshan, A parametrization of bipartite systems based on SU (4) Euler angles. Journal of Physics A: Mathematical and General, 2002. 35(48): p. 10445.

[19] Uchida, G., Geometry of GHZ type quantum states, in Fakulti Fizik. 2013, Universiti Vienna.

[20] Makhlin, Y., Nonlocal Properties of Two-Qubit Gates and Mixed States, and the Optimization of Quantum Computations. Quantum Information Processing, 2002. 1(4): p. 243-252.

[21] Idrus, B., et al., Correlations in a chain of three oscillators with nearest neighbour coupling. Journal of Modern Optics, 2010. 57(7): p. 576-581.

[22] Zhu, X.-N. and S.-M. Fei, Lower bound of concurrence for qubit systems. Quantum Information Processing, 2013: p. 1-9.

[23] Carlos Sabín and Guillermo García-Alcaine, A classification of entanglement in three-qubit systems. The european physical journal D, 2008. 48(3): p. 435-442.

[24] Lohmayer, R., et al., Entangled Three-Qubit States without Concurrence and Three-Tangle. Physical Review Letters, 2006. 97(26): p. 260502. 